\title{
LIDAR CALIBRATION CENTRE
}

\author{
Gelsomina Pappalardo ${ }^{1}$, Volker Freudenthaler ${ }^{2}$, Doina Nicolae ${ }^{3}$, Lucia Mona ${ }^{1}$, Livio Belegante ${ }^{3}$, \\ Giuseppe D'Amico' \\ ${ }^{1}$ Consiglio Nazionale delle Ricerche, Istituto di Metodologie per l'Analisi Ambientale (CNR-IMAA), Tito \\ Scalo, Potenza, Italy \\ ${ }^{2}$ Ludwig Maximilians University, Meteorological Institute Munich in German \\ ${ }^{3}$ National Institute of R\&D for Optoelectronics, Magurele, Romania
}

\begin{abstract}
This paper presents the newly established Lidar Calibration Centre, a distributed infrastructure in Europe, whose goal is to offer services for complete characterization and calibration of lidars and ceilometers. Mobile reference lidars, laboratories for testing and characterization of optics and electronics, facilities for inspection and debugging of instruments, as well as for training in good practices are open to users from the scientific community, operational services and private sector. The Lidar Calibration Centre offers support for trans-national access through the EC HORIZON2020 project ACTRIS-2.
\end{abstract}

\section{INTRODUCTION}

Lidars and ceilometers are based on the same principle, but significantly different from the engineering point of view, as they are meant for different applications. However, calibration is one common challenge. Lidars are more complex in terms of laser power, number of detection channels and therefore of the optics and electronics. This helps to obtain more quantitative data products [1] (e.g. backscatter and extinction profiles at several wavelengths, lidar ratios, Angstrom exponents and particle depolarization ratios), however with an uncertainty that has to be well documented. An important contribution to the data uncertainty comes from the instrument's imperfections, which are generally difficult to quantify. Each optical or electronic component has an impact, but they also interact at a certain level, and depend on the environmental conditions. For example, the transmission of the interference filters at the laser wavelength changes with temperature, while the dynamic range of the whole system varies with the laser-telescope alignment, also depending on the temperature.

Lidars operate in different configurations and designs. The quality of the data products depends strongly on the instrument performance and calibration, but a direct calibration is not possible because they measure remotely in ambient conditions. Coupling of the system with the atmosphere is also a disturbing factor. Due to the complexity of the instrument and large variety of designs, systematic errors are difficult to be objectively assessed. On the other hand, data uncertainty is underestimated if systematic errors are not included.

Worldwide, there are several lidar networks, regional or global in scope. EARLINET (the European Aerosol Research Lidar NETwork) was founded in 2000 as a research project for establishing a quantitative, comprehensive, and statistically significant database for the horizontal, vertical, and temporal distribution of aerosols on a continental scale [2]. Since then, EARLINET has collected and published more than 45000 vertical profiles of aerosols [3],[4],[5],[6],[7]. GALION (the Global Aerosol LIdar Network) strives to determine the vertical component of the spatiotemporal distribution of aerosol properties related to climate forcing and air quality, in a network of ground-based lidar stations [8]. To achieve this goal, homogeneous data products are required. This homogeneity can only be achieved by implementing common calibration procedures and making use of the existing calibration facilities.

Calibration is possible by direct comparison with reference instruments off-and-on, but has to be regularly checked by means of quality assurance (QA) tests applied by properly trained staff.

\section{LICAL INFRASTRUCTURE}

The Lidar Calibration Centre (LiCal) is a multiinstallation facility, offering a wide range of possibilities to test and calibrate lidars and ceilometers, starting from the characterization and 
optimization of single components to the assessment of the whole system's performance. It combines a series of direct and remote techniques, involving both the equipment and the operators, to ensure a proper evaluation, maintenance, calibration and operation. Each of the seven installations has a specific role in this process.

The Lidar Calibration Training Laboratory (LiCalTrain) is operated by the National Institute of R\&D for Optoelectronics in Bucharest, Romania. The infrastructure consists of a multiwavelength depolarization Raman lidar, a scanning depolarization UV lidar, a tropospheric ozone lidar and complementary instrumentation (Scanning Microwave radiometer, C-ToF Aerosol Mass Spectrometer, Aerosol Chemical Speciation Monitor, Aethalometer, Aerodynamic Particle Sizer, Integrating nephelometer), as well as dedicated software tools to test, characterize, calibrate and validate lidar measurements. It is ideal to train the operators how to apply regular check-ups and maintain the calibration.

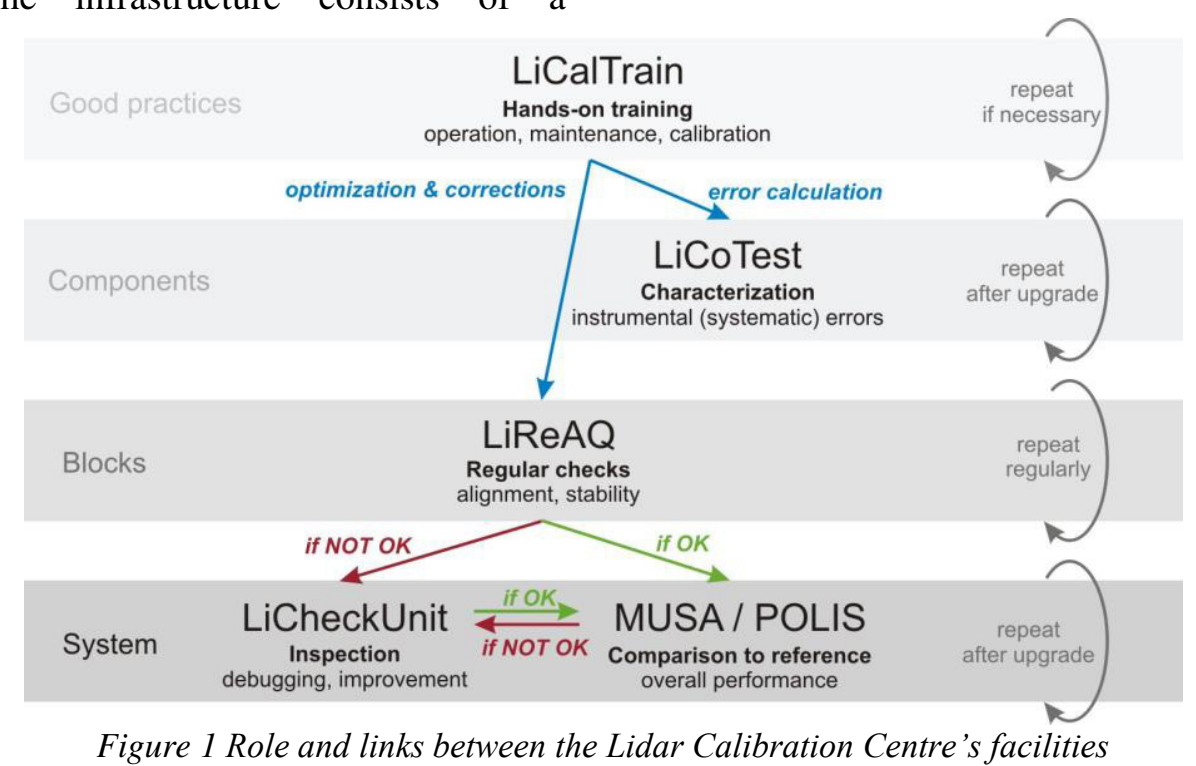

The mobile reference lidar POLIS operated by the Meteorological Institute at Ludwig Maximilians University in Munich, Germany, measures at two elastic wavelengths $-355 \mathrm{~nm}$ and $532 \mathrm{~nm}$ and their $\mathrm{N}_{2}$-Raman shifted wavelengths - $387 \mathrm{~nm}$ and $607 \mathrm{~nm}$, and linear depolarisation ratio at $532 \mathrm{~nm}$ and $355 \mathrm{~nm}$. It is portable and can be easily moved to the user's site by air cargo to perform side-by-side comparison. It is the only reference lidar with depolarization at $355 \mathrm{~nm}$.

The reference lidar MUSA operated by the Institute of Methodologies for Environmental Analysis - National Research Council, in Potenza, Italy is a multi-wavelength depolarization Raman with 3 elastic - 1064, 532, $355 \mathrm{~nm}, 2$ Raman 607, $387 \mathrm{~nm}$, and linear depolarization ratio at 532 $\mathrm{nm}$. It is the only reference instrument operating simultaneously 3 elastic and 2 Raman channels. The system can be moved to the user site (if the user's lidar is fixed) or can be operated at the host for longer intercomparisons.
The availability of the two reference systems provides a good geographical coverage and reduces time delays between demand and supply.

The Lidar Components Testing Laboratory (LiCoTest) hosted by the National Institute of $\mathrm{R} \& \mathrm{D}$ for Optoelectronics has a long expertise in characterizing laser beams (pulse width, Gaussian parameters, beam shape) and optical components (angles between plane surfaces, refractive index, surface characterization), using power and energy meters, Tektronics oscillioscopes, Beam Profiler with $\mathrm{M}^{2}$ option, BeamScope, goniometer and interferometer. LiCoTest also covers spectroscopic ellipsometric measurements and spectral characterization of interferential filters (bandwidth, transmission, blocking), dichroic beam splitters and mirrors, and characterization of the aging process with the Perkin Elmer UVVIS NIR Spectrometer. Electronic modules are characterized and tested with Agilent and Tektronics pulse/form generators and analysers. 
This installation delivers full characterization of the optical and electronic components of the lidar transmitter and receiver, which is essential when assessing the instrumental systematic errors.

The Lidar Remote Quality Assurance (LiReQA) and the Lidar Check-up Unit (LiCheckUnit) are operated by the Meteorological Institute at Ludwig Maximilians University

\section{The Lidar Remote Quality Assurance} (LiReQA) operates dedicated software tools (raytracing software for analyzing the transmitter and receiver optics, lidar polarization analysis software, lidar radiation power budget analysis software, analysis of test measurements specialized for individual lidar setups) to perform fault diagnosis of any lidar system.

The Lidar Check-up Unit (LiCheckUnit) is a portable unit (laser power meter, polarization analysis instruments for inspection of transmitter and receiver optics, adapted instruments for checking the alignment of the lidar system, e.g. CCD camera \& special optics adapted to the lidar system, lidar pulse simulator for inspection of the detection electronics) which can be used at the user site to inspect and debug the instrument.

LiCal has been set up following a long process for standardization and harmonization of calibration procedures within EARLINET community. Mature techniques were picked up by LiCal and are now offered to the users as services, while research and technological development continues in collaboration with EARLINET and ACTRIS. The techniques and the methodologies used by LiCal emerged from EARLINET's scientific and technological activities.

The services offered by the LiCal infrastructure are part of a complete characterization and optimization of lidar instruments. While the characterization of basic components (laser, optical and electronic parts) is performed at LiCoTest (laser beam characterization, optical and spectral characterization of lenses, filters, mirrors and fiber optics, spectroscopic ellipsometry measurements, polarization measurements, paraxial analysis of optical systems, electronic modules synchronization, stability, response time/speed), the assessment of the overall system's performance is made by a side-by-side comparison with POLIS or MUSA, which are both EARLINET reference mobile lidar systems.

Systematic quality assurance of the optical and electronic chains and fault diagnosis is performed by LiReQA, while users are trained at LiCalTrain to apply the specific tests. Inspection, debugging and optimization of lidars at their site is performed by LiCheckUnit.

\section{RESULTS}

The various installations of $\mathrm{LiCal}$ have offered in the last years a significant number of accesses within EARLINET, ACTRIS (Aerosols, Clouds and Trace gases Research Infrastructure Network), ESA-CEOS, Initial Training for atmospheric Remote Sensing network (ITARS), as well as for the master and doctoral programs at the Romanian, Italian and German Universities, and national research programs. On average, LiCal installations have hosted about 48 projects (intercomparisons, sample analysis, training, system inspection) and 1260 access days per year.

Within the frame of EARLINET and ACTRIS, LiCal has been serving the scientific community (about 20 active lidar stations, regular access), the user community (several lidar/ceilometer operators belonging to weather services and aviation) and the private sector (about 3 companies / developers and integrators).

LiCal was also involved in intercomparison (SLiCE2000, EARLI09, SPALI10, SOLI10, ROLI10, AQUILI12, NALI13, LELI13) and scientific campaigns (ACTRIS Summer 2012 Campaign, Charmex EARLINET 72h exerciseJul. 2012, EMEP summer and winter campaignJun.-Jul. 2012 and Jan.-Feb. 2013, Charmex 2013, CHARADMExp 2014).

This activity strongly contributed to increase the number of available quality assured EARLINET data as demonstrated by the large number of publications collected in the last years (a complete list is reported at www.earlinet.org). In particular, EARLINET data have been recently used for aerosol characterization in terms of optical and microphysical properties in conjunction with in situ measurements and transport models (e.g. [9],[10],[11],[12]).

The long-term EARLINET quality assured database is the basis for climatological studies, 
systematic comparison with models and satellite observations ([13],[14]). New products were derived as further outcome of this calibration activity, e.g the calibrated linear volume depolarization ratio [15], the particle linear depolarization ratio [16]. At the same time, more and more lidars were upgraded with Raman and depolarization channels, so the number of accesses to LiCal increased as well.

\section{CONCLUSIONS}

A Lidar Calibration Centre was recently set-up in Europe. Its task is to provide complete calibration of lidars and ceilometers, and to provide proper tools to assess the instrumental biases. It is a distributed infrastructure, relying on state-of-theart laboratories for testing and characterization of optical and electronic components and blocks, on mobile reference instruments, and on experienced experts for each topic. Training in good practices in lidar operation and maintenance is also provided. The Lidar Calibration Centre serves ACTRIS (Aerosol, Clouds and Trace gases Research InfraStructure), but also other worldwide networks and operators, system and components manufacturers. Open access and support to the users is provided through the HORIZON2020 contract ACTRIS-2.

\section{ACKNOWLEDGEMENT}

This work is supported by the EC HORIZON2020 project ACTRIS-2, contract INFRAIA-1-20142015 no. 654109.

\section{REFERENCES}

[1] Gasteiger, J. et al., 2011: Volcanic ash from Iceland over Munich: mass concentration retrieved from ground-based remote sensing measurements Atmos Chem Phys, 11, 2209-2223.

[2] Pappalardo et al., 2014: EARLINET: towards an advanced sustainable European aerosol lidar network, Atmos. Meas. Tech., 7, 2389-2409.

[3] The EARLINET publishing group 2000-2010, (2014)doi:10.1594/WDCC/EN_all_measurement s_2000-2010.

[4] The EARLINET publishing group 2000-2010, (2014b)doi:10.1594/WDCC/EN_Climatology_20 00-2010.

[5] The EARLINET publishing group 2000-2010, (2014)doi:10.1594/WDCC/EN_Calipso_20062010.
[6] The EARLINET publishing group 2000-2010, (2014)doi:10.1594/WDCC/EN_VolcanicEruptio n_2000-2010.

[7] The EARLINET publishing group 2000-2010, (2014)doi:10.1594/WDCC/EARLINET_Saharan Dust_2000-2010.

[8]Bösenberg J., Hoff R. et al., 2007: Plan for the implementation of the GAW Aerosol Lidar Observation Network GALION, GAW Report No. 178, Hamburg, Germnay

[9] Pappalardo, G. et al., 2013.: Four-dimensional distribution of the 2010 Eyjafjallajökull volcanic cloud over Europe observed by EARLINET, Atmos. Chem. Phys., 13, 4429-4450.

[10] Sicard, M. et al., 2012: Monitoring of the Eyjafjallajökull volcanic aerosol plume over the Iberian Peninsula by means of four EARLINET lidar stations, Atmos. Chem. Phys., 12, 311531130.

[11] Wagner, J. et al., 2013: Evaluation of the Lidar/Radiometer Inversion Code (LIRIC) to determine microphysical properties of volcanic and desert dust, Atmos. Meas. Tech., 6, 17071724.

[12] Nicolae, D. et al., 2013: Characterization of fresh and aged biomass burning events using multiwavelength Raman lidar and mass spectrometry, J. Geophys. Res. Atmos., 118, 2956-2965.

[13] Mona, L. et al., 2012: Lidar Measurements for Desert Dust Characterization: An Overview, Advances in Meteorology, 2012, 356265, 1-36.

[14] Pappalardo, G. et al., 2010: EARLINET correlative measurements for CALIPSO: first intercomparison results, J. Geophys. Res., doi:10.1029/2009JD012147.

[15] Bravo-Aranda, J. A. et al., 2013: Analysis of lidar depolarization calibration procedure and application to the atmospheric aerosol characterization, Int. J. Rem. Sens., 34 (9-10), 3543-3560.

[16] Freudenthaler, V. et al., 2009: Depolarizationratio profiling at several wavelengths in pure Saharan dust during SAMUM 2006, Tellus B, 61, 165-179. 